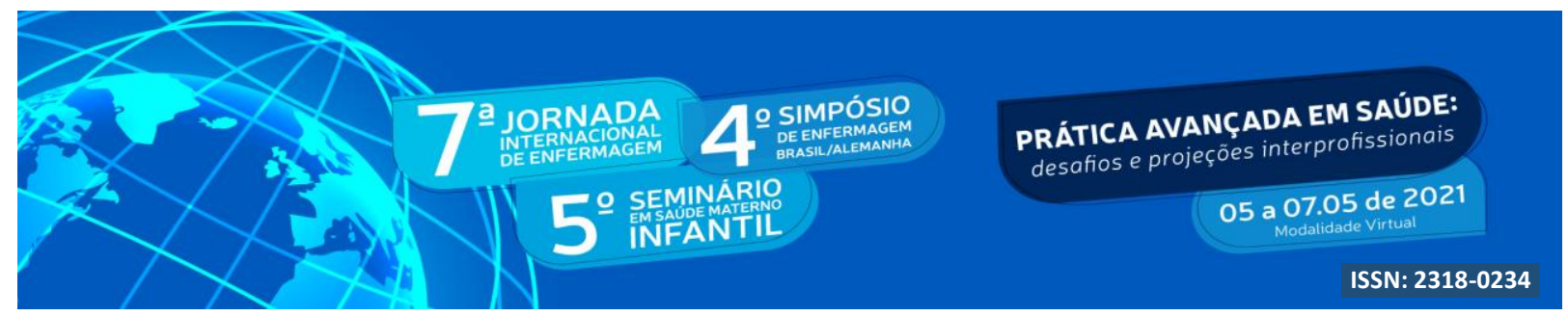

DOI: http://doi.org/10.48195/jie2021-055

\title{
RELATO DE EXPERIÊNCIA SOBRE A REALIZAÇÃO DE UM PROCEDIMENTO OPERACIONAL PADRÃO EM CURATIVO. ${ }^{1}$
}

\author{
Isadora Vasconcelos ${ }^{2}$; Adriélli Balconi ${ }^{3}$; Alielly Camargo ${ }^{4}$; Sheila Lucas da Silveira \\ Tavares ${ }^{5}$.
}

\section{RESUMO}

Este trabalho tem por objetivo demonstrar a experiência de realizar um guia técnico sobre curativos, o qual permitirá a padronização da técnica. Trata-se de uma pesquisa-ação vivenciada no estágio observacional nas Unidades Básicas de Saúde no Município de Bagé-RS. Através das visitas, observamos as dificuldades na realização do procedimento e negligência com o uso de Equipamentos de Proteção Individual (EPIS) pelos profisssionais de enfermagem. Ainda, o diálogo com os enfermeiros evidenciou a necessidade de materiais para padronizar a assistência. Por conseguinte, nota-se a relevância no Procedimento Operacional Padrão que elaboramos. Sendo assim, espera-se que essa pesquisa proporcione à equipe uma preparação e autonomia que melhora a realização do procedimento.

Palavras-chave: Curativos; Enfermagem; POP.

\begin{abstract}
This work aims to demonstrate the experience of carrying out a technical guide on dressings, which will allow the standardization of the technique. It is an action research experienced in the observational stage in the Basic Health Units in the Municipality of Bagé-RS. Through visits, we observe the difficulties in performing the procedure and neglect with the use of Personal Protective Equipment (EPIS) by nursing professionals. Still, the dialogue with nurses showed the need for materials to standardize care. Therefore, it is noted the relevance in the Standard Operating Procedure that we elaborate. Therefore, it is expected that this research will provide the team with preparation and autonomy that improves the performance of the procedure.
\end{abstract}

Key Words: Dressings; Nursing; POP.

\footnotetext{
${ }^{1}$ Relato de experiência. Bagé. RS.

${ }^{2}$ Estudante de Enfermagem. URCAMP. Bagé. RS. E-mail: amaralvasconcelosisadora@gmail.com

${ }^{3}$ Estudante de Enfermagem. FISMA. Santa Maria. RS. E-mail: adriidalgobalconi@ gmail.com

${ }^{4}$ Estudante de Enfermagem. URCAMP. Bagé. RS. E-mail: aliellycamargo2504@gmail.com

${ }^{5}$ Orientador. Enfermeiro. Professor. URCAMP. Bagé. RS. E-mail: Sheilatavares@ urcamp.Edu.be
} 


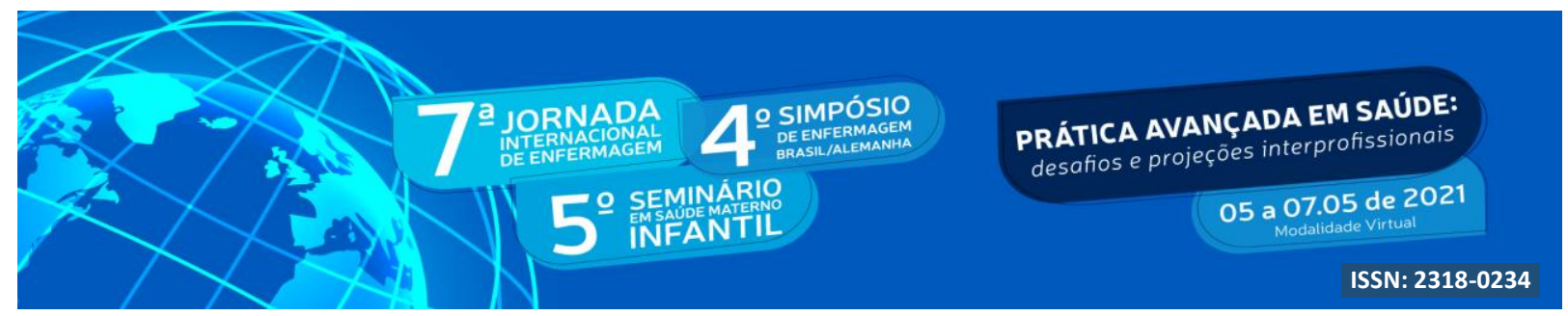

\section{INTRODUÇÃO}

Procedimento Operacional Padrão (POP) é um documento que viabiliza procedimentos seguros embasados em evidências científicas, onde descreve cada atividade de forma padronizada e sistematizada o que possibilita a confiabilidade da assistência. O POP deve ser entendido como uma sistematização dos processos, permitindo à equipe ordenar a execução de determinado procedimento garantindo resultados homogêneos, permite a absorção de informações, melhorando o desempenho profissional e promovendo o conhecimento para a melhoria na prática (PEREIRA et al., 2017).

Desta forma a padronização dos serviços de enfermagem se faz essencial, uma vez que essa é uma ferramenta importante no processo gerencial, pois deixa as ações mais homogêneas, reduzindo a falta de atenção, permitindo a realização dos serviços de forma organizada, orientada e segura, estimulando a criatividade e a participação de toda a equipe (MENDES, 2016).

Este contém instruções aprofundadas, que orientam na execução de determinada atividade, assim proporciona a uniformização e favorece na qualidade, eficiência e eficácia das ações evitando que ocorra desvio na execução das atividades. Neste contexto o enfermeiro realizando a capacitação por meio do POP proporcionará o preparo da equipe na condução do curativo, profissionalismo, credibilidade, diminuição dos gastos de material, melhora no processo de trabalho e atendimento de qualidade aos pacientes e equipe (PEREIRA et al., 2019).

Portanto, ao considerar as muitas atividades desempenhadas pelos enfermeiros, a execução do curativo pode ser delegada ao técnico de enfermagem, desde que sob supervisão e acompanhamento do enfermeiro. Salienta-se a importância de se utilizar o instrumento POP como ferramenta para a capacitação e formação dos profissionais da enfermagem. Esse deve ser capaz de proporcionar uma efetiva e adequada sistematização da assistência de enfermagem (HORTA; RUFINO; NEVES, 2018).

A construção do documento é de responsabilidade do gestor do processo e este poderá designar um colaborador para realizar tal atividade. São considerados apropriados para uso na 


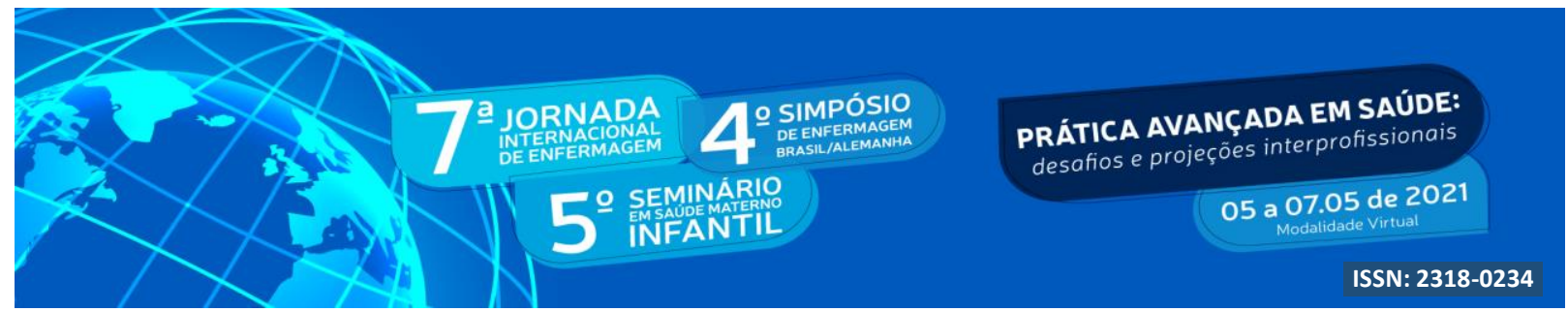

instituição apenas os documentos eletrônicos disponibilizados no portal corporativo institucional (intranet) e os documentos impressos que contenham assinatura e carimbo de um representante do Setor de Gestão da Qualidade e da Segurança do Paciente juntamente com carimbo de cópia controlada (NEPEN, 2019).

Para garantir que apenas as versões atuais e relevantes dos documentos estejam disponíveis, a impressão de documentos só é permitida com autorização e de forma controlada. Por tanto, as versões eletrônicas devem ser disponibilizadas com impedimento de edição e impressão. Os destinos das cópias físicas e eletrônicas devem ser registrados em planilha de controle para permitir a rastreabilidade e recolhimento das versões obsoletas (NEPEN, 2019).

Em suma, a criação do protocolo, realizada por estudantes enfermagem busca proporcionar assistência ao paciente portador de feridas, buscando por qualidade e humanização durante o tratamento, seguindo as diretrizes propostas pelo SUS para assim obter sucesso com o atendimento, e por consequência melhorar as estatísticas do país.

\section{OBJETIVO}

Demonstrar a criação de um Procedimento Operacional Padrão (POP), tento em vista o aumento da prevalência de feridas se fez necessário buscar conscientizar e padronizar a assistência, levando os profissionais de saúde a realizarem uma técnica de curativo adequada que ajudará o organismo estimulando a cicatrização, prevenindo contaminações e infecções.

\section{METODOLOGIA}

O Relato de Experiência é um conhecimento que se transmite com aporte científico, descritivo em forma de narrativa, que o autor narra através da escrita um acontecimento vivido, com o uso das observações subjetivas (sentimentos/impressões) e/ou objetivas (observação participante, por exemplo) (GROLLMUS; TARRÉS, 2015).

Este trabalho surgiu da iniciativa de contar uma experiência vivida na Secretaria Municipal de Saúde de Bagé, na região da Campanha do Rio Grande do Sul, entre os meses 


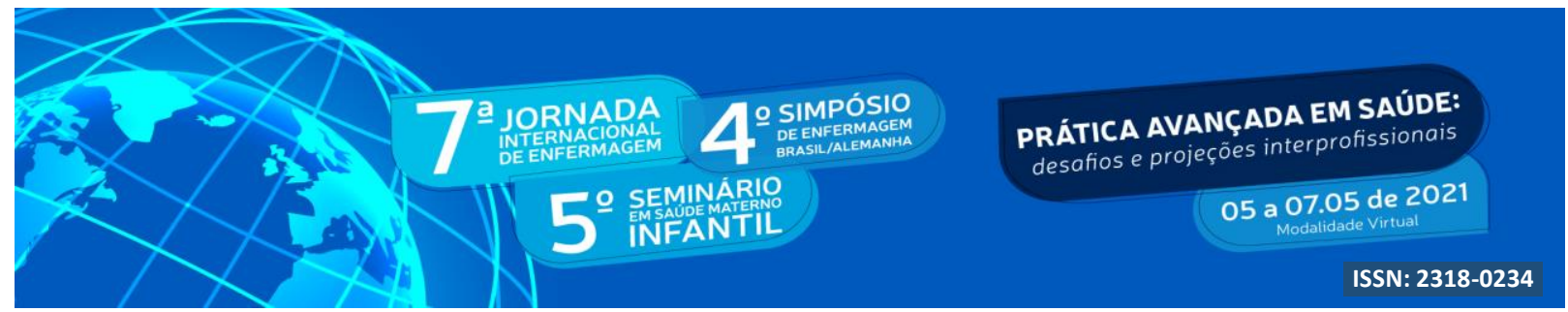

de setembro a novembro de 2020, conforme necessidade encontrada num período observacional de estágio em duas Unidades Básicas de Saúde daquela cidade. As análises aconteceram com profissionais de enfermagem que prestavam assistência de curativos nas segundas-feiras do mês de novembro no ano de 2020 entre os períodos manhã e tarde.

\section{RESULTADOS E DISCUSSÃO:}

Os resultados das discussões direcionaram-se para observar a conduta profissional na realização de curativos.

Ambas as Unidades possibilitaram diversas visualizações de seis procedimentos em relação aos curativos, as idades dos pacientes variaram entre 60 e 104 anos, onde alguns dos enfermos se deslocavam em direção a UBS e outros solicitavam a visita domiciliar para a realização dos cuidados.

As considerações após o acompanhamento para a execução dos curativos possibilitaram a observação de que os profissionais possuem dificuldades em desempenhar as etapas corretas dos procedimentos e, infelizmente, muitas vezes são negligentes ao uso de EPI,'s.

Segundo PERUZZO et al.; 2008 deve ser mantido os seguintes princípios específicos para realização dos curativos: lavagem das mãos, manipular material estéril com luvas estéreis, não falar ao manipular ferida e material estéril, dispor o material: evitar contaminação, abrir materiais com técnica adequada: manter esterilidade, orientar o cliente para: não tocar na ferida, não falar ou tocar nos materiais, preparar o ambiente: janelas, portas, seleção recurso adequado, limpar ferida com S.F. 0,9\%, limpeza: do menos contaminado para o mais contaminado, zona mais contaminada da ferida: parte de baixo, não tocar na lesão; usar uma gaze de cada vez com tampão rotativo, remover secreções.

Vivenciamos as orientações realizadas aos pacientes, os acompanhamentos prestados pela equipe de saúde e a partir desse momento, consideramos as particularidades e as atribuições de cada profissional. A primeira avaliação e condutas iniciais serão realizadas pelo enfermeiro.

A equipe realizará o cuidado compartilhado, porém salientamos a importância dos 


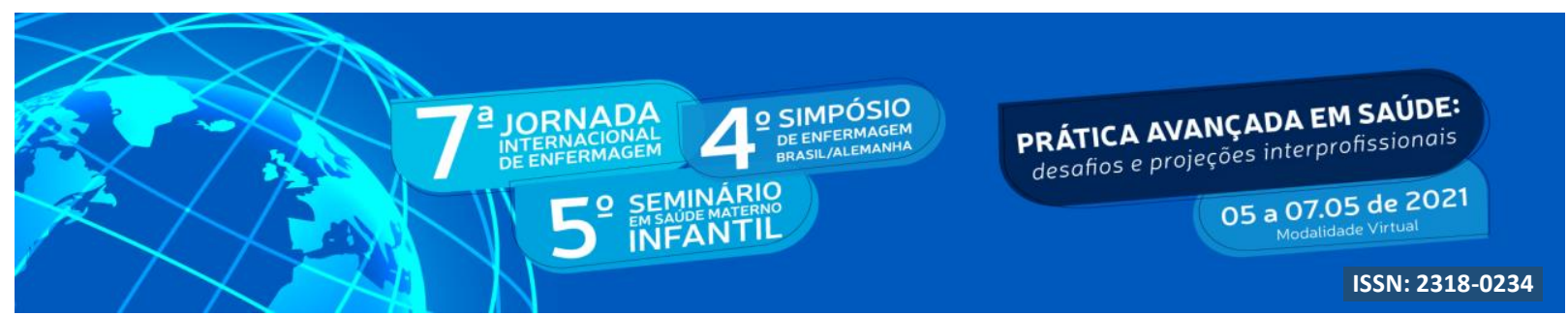

pacientes serem informados sobre os fatores que afetam na sua cicatrização, alguns destes são de acordo com a Prefeitura de Florianópolis (2019):

Idade, pois, à medida que a idade avança o ritmo do metabolismo celular diminui assim apresentando maior risco para desnutrição pela má absorção;

Patologias associadas/comorbidades como: diabetes, doenças imunológicas, câncer, insuficiências vasculares, entre outras, interferem no processo de cicatrização;

Alimentação adequada para evitar deficiência de proteínas, calorias, vitamina A e E, zinco, arginina e glutamina alteram a cicatrização, assim como a desnutrição e a obesidade;

Infecção, pois, ela retarda o processo de cicatrização por competir com a ferida por nutrientes.

Um dos dias marcantes de nossa vivência foi um desbridamento instrumental que foi realizado no leito para remover tecido necrótico. Ocorrendo a retirada do tecido necrótico com abordagem conservadora, isto é, acima do tecido viável, sem causar dor ou sangramento (GIRONDI et al.; 2018).

Os diálogos com os profissionais constituíram relatos onde abordaram a falta de materiais atualizados para instruir-se quando necessário e, consequentemente isso melhoraria os saberes, além de estimular o desenvolvimento do raciocínio clínico que é muito importante para a tomada de decisão e fundamental no cotidiano da Enfermagem.

Salienta-se que esta colocação foi de extrema importância para a criação do nosso POP, e este diálogo também foi nos colocado assuntos que eles achavam de relevância que estivessem presente no mesmo, entre eles:

Classificação das feridas: Segundo COSTA et al.; (2019) as feridas se classificam quanto sua etiologia, quanto ao seu tempo de duração e de acordo com o grau de contaminação.

Classificação etiológica: Cirúrgicas: produzidas por instrumentos cirúrgicos e tem finalidade terapêutica. Traumáticas: produzidas acidentalmente, podendo ser causadas por agentes: mecânicos (pregos, facas, barra de ferro), agentes físicos (frio, calor, radiação, eletricidade) e químicos (cosméticos, soda cáustica, ácido). Ulcerativas: lesões escavadas, circunscritas na pele, podendo ter profundidade variável, atingir apenas camadas superficiais ou ultrapassar essas e atingir até os músculos (COSTA et al.; 2019). 


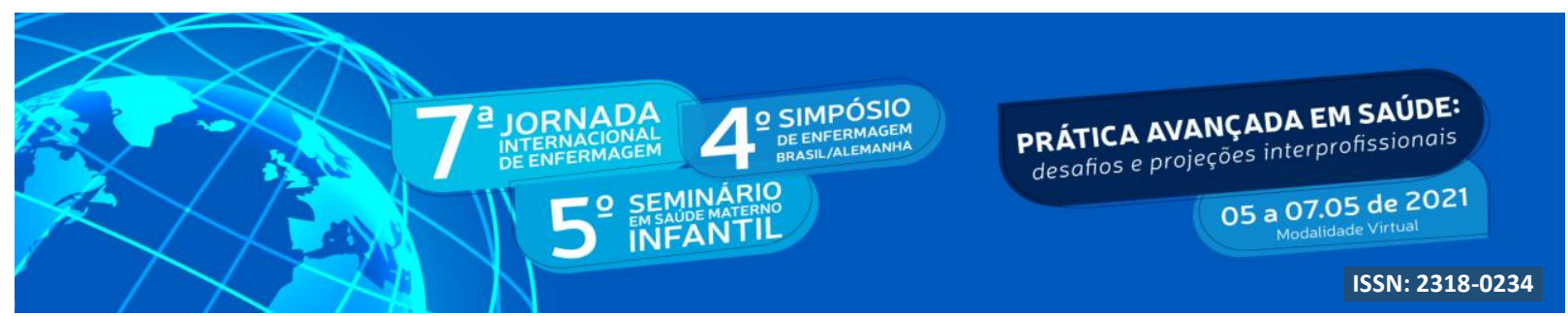

Classificação de acordo com o tempo: Feridas Agudas: com boa resposta ao tratamento, em geral com cicatrização rápida e sem complicações. Feridas Crônicas: cicatrização lenta, com complicações no processo e na sequência da reparação tecidual. (COSTA et al.; 2019).

Classificação quanto ao grau de contaminação: Feridas Limpas: sem sinais de infecção, feitas e mantidas em condições assépticas. Feridas Potencialmente Contaminadas/Limpa-contaminadas: feridas com menos de 6 horas, sem contaminação significativa ou contaminadas, porém de maneira controlada ou premeditada. Feridas Contaminadas: feridas com mais de 6 horas ou em que a técnica asséptica não foi realizada de maneira adequada, porém ainda sem processo infeccioso. Feridas Infectadas: com sinais claros de infecção/reação inflamatória (edema, calor, rubor e dor) (COSTA et al.; 2019).

Classificação dos curativos: sendo eles os semi-oclusivos que é um curativo absorvente, geralmente é utilizado em feridas cirúrgicas, drenos, feridas exsudativas, absorvendo o exsudato e isolando-o da pele adjacente saudável. Curativo oclusivo, não permite a entrada de ar ou fluídos, atua como barreira mecânica, impede a perda de fluídos, promove isolamento térmico, veda a ferida, a fim de impedir enfisema, e formação de crosta. Curativo compressivo, é utilizado para reduzir o fluxo sanguíneo, promover a estase e ajudar na aproximação das extremidades da lesão e curativos abertos realizados em ferimentos que não há necessidade de serem ocluídos. Feridas cirúrgicas limpas após 24 horas, cortes pequenos, suturas, escoriações, etc são exemplos deste tipo de curativo (KREMER et al.; 2017).

As vivências proporcionaram aprendizados em relação aos profissionais de saúde, em vista que muitos dedicam constantemente os dias nas UBS e necessitam enfrentar os empecilhos diários, como a falta de materiais sofisticados para melhoria na qualidade do atendimento, a falta de valorização, respeito e compreensão. Espera-se que ao longo dos anos a enfermagem seja reconhecida e valorizada.

Acreditamos que o trabalho contribuiu para um crescimento como acadêmicos e futuros profissionais, buscamos elaborá-lo de forma didática e informativa, para que pudéssemos acrescentar ainda mais conhecimento à equipe de saúde da cidade de Bagé-RS. 


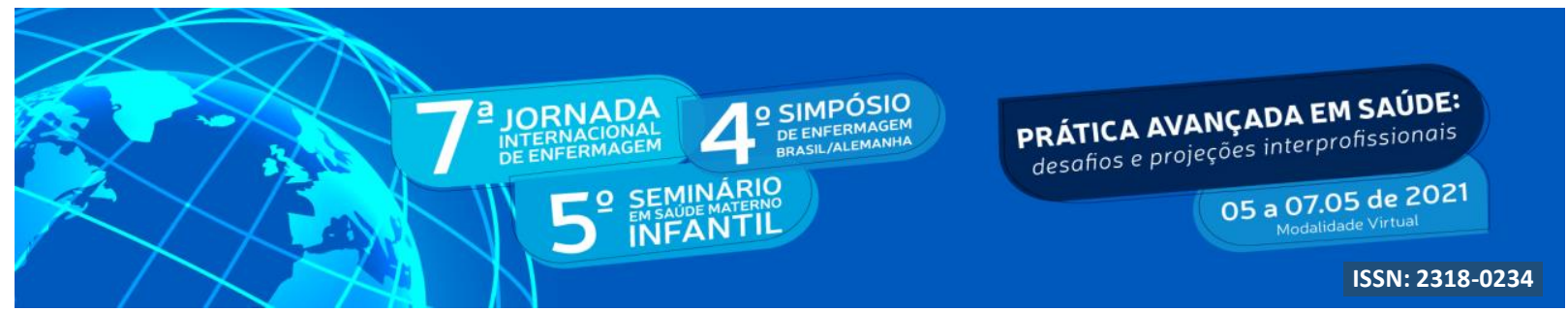

\section{CONCLUSÃO}

Esta experiência possibilitou aprendermos mais sobre pesquisa, buscou-se leituras de artigos, sites, revistas. Percebemos que mesmo havendo descobertas e inovações no campo de cicatrização e curativos, o avanço é necessário devido aos inúmeros déficits e complicações encontradas no tratamento, além da acessibilidade aos avanços já evidenciados, a fim de tornar as tecnologias simples e baratas tão eficazes.

Esperamos que o trabalho venha a ajudar e acrescentar conhecimento e informações proporcionando uma melhor eficiência no atendimento a pessoas com feridas.

Desejamos que as capacitações por meio do POP venha proporcionar o preparo da equipe na condução do curativo, diminuição dos gastos de material, melhora no processo de trabalho e atendimento de qualidade aos pacientes e equipe. Almejamos também um maior reconhecimento da importância e a autonomia da equipe de enfermagem no tratamento de feridas, fazendo com que a enfermagem seja valorizada dentro da equipe multiprofissional.

\section{REFERÊNCIAS}

COSTA, A. P; CAMPOS, E. R; MARIANA, P. E; VIEIRA, E.L.B; ARAÚJO, J.M.A; CHAVES, L.G.S.M; OLIVEIRA, M.R. PROTOCOLO DE RECONHECIMENTO E TRATAMENTO DE FERIDAS NA ATENÇÃo PRIMÁRIA. Centro Universitário São Lucas. Porto Velho, 2019. Disponível em: <http://repositorio.saolucas.edu.br:8080/xmlui/bitstream/handle/123456789/3369/Aline\%20Pi can\%C3\% A7o\%20Costa\%20et \%20al\%20.\%20Protocolo\%20de\%20reconhecimento\%20e\%2 0tratamento\%20de\%20feridas\%20na\%20aten\%C3\%A7\%C3\%A3o\%20prim\%C3\%A1ria.pdf ?sequence $=1>$. Acesso em: 08 set, 2020.

GIRONDI, J.B.R; SOLDERA, D; EVARISTO, S.M; LOCKS, M.O.H; AMANTE, L.N; VIEIRA, A. $S$. Desbridamento de feridas em idosos na atenção primária em saúde. Enfermagem em Foco, [S.1.], v. 10, n. 5, maio 2020. ISSN 2357-707X. Disponível em: <http://revista.cofen.gov.br/index.php/enfermagem/article/view/2669/628>. Acesso em: 14 set. 2020 .

GROLLMUS, NICHOLAS S.; TARRÈS, JOAN P. Relatos metodológicos: difractando experiências narrativas de investigación. Fórum Qualitative Social Research, v. 16, n. 2, 2015. Disponível em: $<$ file:///C:/Users/Particular/Downloads/2207-9561-1-PB\%20(1).pdf>. 


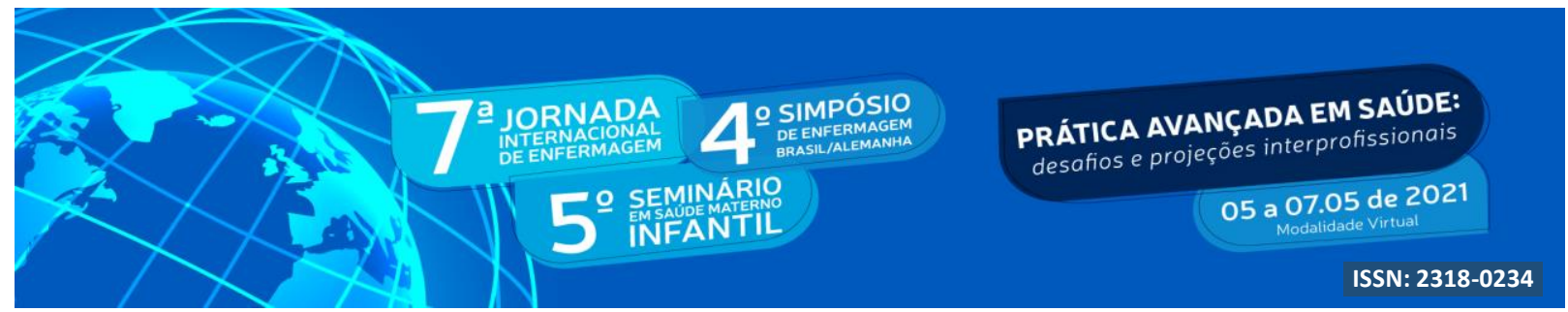

HORTA, I. P. M; RUFINO, M. H. R. O; NEVES, R. S. VALIDAÇÃo DE PROCEDIMENTO OPERACIONAL PADRÃO NO CUIDADO À PESSOA COM FERIDAS. Revista Feridas, v. 34, n. 6, p. 1164-1171, out. 2018. Disponível em: $\langle$ http://www.revistaferidas.com.br/revistas/ed34/pg20.pdf〉. Acesso em: 03 set. 2020.

KREMER, D. W; Prudente, J. A. B; Marques, R; Flores, M.R. CUIDADOS COM A INTEGRIDADE CUTÂNEA. EBSERH- Hospitais Universitários Federais. v.2, p.1-14, nov. 2017. Disponível em: 〈http://www.hu.ufsc.br/pops/pop-externo/download?id=205>. Acesso em 03 set, 2020.

MENDES, V. A. POP- PROCEDIMENTO OPERACIONAL PADRÃO EM SERVIÇOS $<$ http://repositorio.unis.edu.br/bitstream/prefix/527/1/POPS\%20\%E2\%80\%93\%20PROCEDI MENTO\%20OPERACIONAL\%20PADR\%C3\%830\%20EM\%20SERVI\%C3\%870\%20DE \%20EMERG\%C3\%8ANCIA.pdf> Acesso em: 03 set. 2020

NEPEN UFSC. ELABORAÇÃO E CONTROLE DE DOCUMENTOS INSTITUCIONAIS. $\quad$ v.2, $\quad$ p. $1-\quad 30$, jul. 2019. Disponível em: < www2.ebserh.gov.br/documents/2016343/4699516/Norma_de_Elaboracao_e_Controle_de_D ocumentos_v.2 01.08.19.pdf/6443dcd1-56e2-486f-a051-36051fdca948> . Acesso em: 03 set. 2020

PEREIRA J. F. S; Dezoti, A. P; Scussiato, L. A. CAPACITAÇÃo PARA EQUIPE DE ENFERMAGEM DE UMA UNIDADE DE SAÚDE SOBRE ASSISTÊNCIA NOS CURATIVOS. Anais do EVINCI -UniBrasil, Curitiba, v.5, n.1, p.342-342, out. 2019. Disponível em: <https://portaldeperiodicos.unibrasil.com.br/index.php/anaisevinci/article/view/5099/3968>. Acesso em: 03 set, 2020.

PEREIRA, L. R; Carvalho, M. F; Santos, J. S; Machado, G. A. B; Maia, M. A C; Andrade, R. D. AVALIAÇÃO DE PROCEDIMENTOS OPERACIONAIS PADRÃO IMPLANTADOS EM UM SERVIÇO DE SAÚDE. Arquivos de Ciências da Saúde, [S.1.], v. 24, n. 4, p. 47-51, dez. 2017. ISSN 2318-3691. Disponível em: $\langle$ http://www.cienciasdasaude.famerp.br/index.php/racs/article/view/840 > Acesso em: 03 set. 2020 .

PERUZZO, S. A; Boller, S; Taube, S.A.M; Soares, F; Stahlhoefer, T. DESENVOLVIMENTO DO PROTOCOLO DE CURATIVO: RELATO DE EXPERIÊNCIA DA DEMANDA DE UM PROJETO DE EXTENSÃO. DADOS ACHADOS NA PESQUISA. Universidade Federal do Paraná. p. 227-231. 2008. Disponível em: $<$ http://www.abeneventos.com.br/2senabs/cd anais/pdf/id212r0.pdf $>$. Acesso em: 08 set, 2020 .

Prefeitura de Florianópolis. Secretaria Municipal de Saúde de Florianópolis. CUIDADO À PESSOA COM FERIDA. Protocolo de Enfermagem, v.6, p.1-98. Florianópolis, jun. de 2019. 


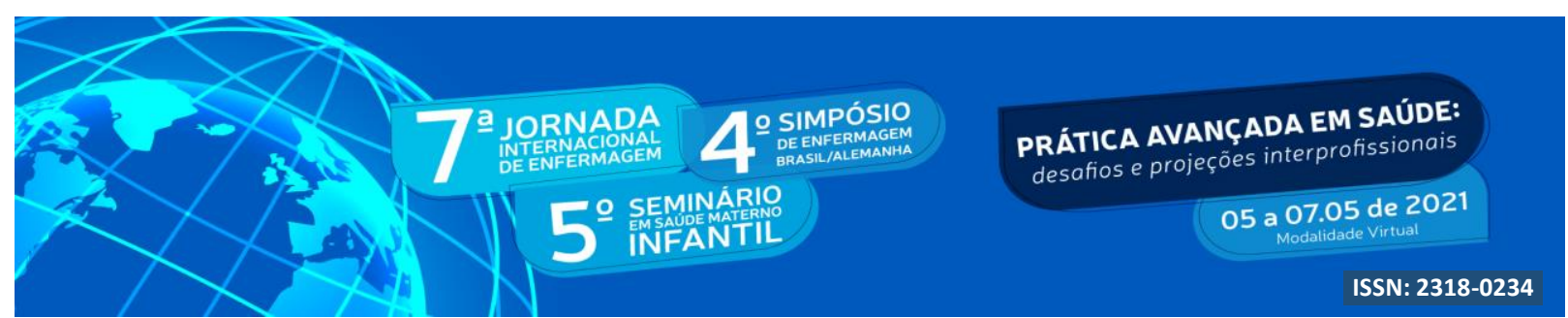

<http://www.pmf.sc.gov.br/arquivos/arquivos/pdf/19_06_2019_14.54.48.a094a8bd10cad8fda d4c98021e73821a.pdf>. Acesso em: 03 set. 2020. 